\title{
Acute haemorrhagic leucoencephalitis with a prolonged clinical course
}

\author{
CHIN-CHANG HUANG, NAI-SHIN CHU, TONG-JONG CHEN, * \\ CHENG-MEI SHAW
}

\author{
From the Departments of Neurology and Pathology, Chang Gung Medical College, Chang Gung Memorial \\ Hospital, Taipei, Taiwan
}

SUMMARY Two cases of pathologically proven acute haemorrhagic leucoencephalitis with clinical features resembling a cerebral tumour are reported. One patient with a left fronto-parieto-temporal mass showed progressive neurological deterioration leading to death in 3 months. Another patient with a right parieto-temporal mass had mild neurological symptoms for one and a half months before complete recovery except for a seizure disorder, following steroid and decompressive therapy. The cases show that acute haemorrhagic leucoencephalitis may not always be rapidly fatal, but may run a prolonged clinical course with recovery.

Acute haemorrhagic leucoencephalitis was first defined pathologically by Hurst ${ }^{1}$ and represents the most severe form of acute disseminated encephalomyelitis. The disease is rare, usually affects young adults and follows an upper respiratory tract infection. ${ }^{1-6}$ The clinical feature is characterised by an abrupt onset of headache, fever, vomiting, nuchal rigidity and disturbance of consciousness, followed by rapidly deepening coma, hemiplegia or quadriplegia and occasional epileptic seizures. ${ }^{1-6}$ The outcome is fatal usually within 10 days to 2 weeks. However, a few survivors have been reported in recent years following decompressive, steroid and dehydration therapy. $^{7-11}$

We have observed two unusual cases of pathologically proven acute haemorrhagic leucoencephalitis that presented as a progressive hemispheric space-occupying lesion and ran a prolonged clinical course of a few months. It appears that there is an atypical and less severe form of acute haemorrhagic leucoencephalitis.

Address for reprint requests: Nai-Shin Chu, MD, Department of Neurology, Chang Gung Medical College, Chang Gung Memorial Hospital, 199 Tung Hwa North Road, Taipei, Taiwan.

Received 17 July 1987 and in revised form 16 December 1987. Accepted 23 December 1987

\section{Case reports}

Case 1 The patient was a 22 year old man who was in his usual health until 20 August, 1983 when he had a generalised convulsion. During the previous month, he had had four episodes of right facial twitching and slurred speech with eye deviation to right. Each episode lasted approximately 5 minutes and occurred about once a week. There was no recent history of vaccination, skin rashes or influenza. On admission he was alert, oriented and afebrile. Blood pressure was $100 / 70 \mathrm{mmHg}$, pulse rate $68 / \mathrm{min}$ and respiratory rate $18 / \mathrm{min}$. The neck was supple. Neurological examination revealed mild motor dysphasia, mild right central facial weakness and pronation of the extended right arm. The deep tendon reflexes were more brisk on the right, but the plantar response was flexor bilaterally. Laboratory studies including complete blood count, sedimentation rate, and liver function tests were normal. Electroencephalography (EEG) disclosed intermittent theta and occasional delta waves over left fronto-central areas. Computed tomography (CT) demonstrated an area of decreased density with heterogenous enhancement in the left fronto-parieto-temporal areas with a mid-line shift. Cerebral angiography showed the mass lesion with abundant vascular stain. Pathological examination of the specimens obtained from the stereotaxic brain biopsy showed perivascular lymphocytic cuffing in the white matter but no tumour tissue was found. A tentative diagnosis of encephalitis was entertained. The patient was treated with dexamethasone and phenytoin. His neurological status became slightly worse despite steroid therapy. There were increasing headache, motor dysphasia, right facial and arm 

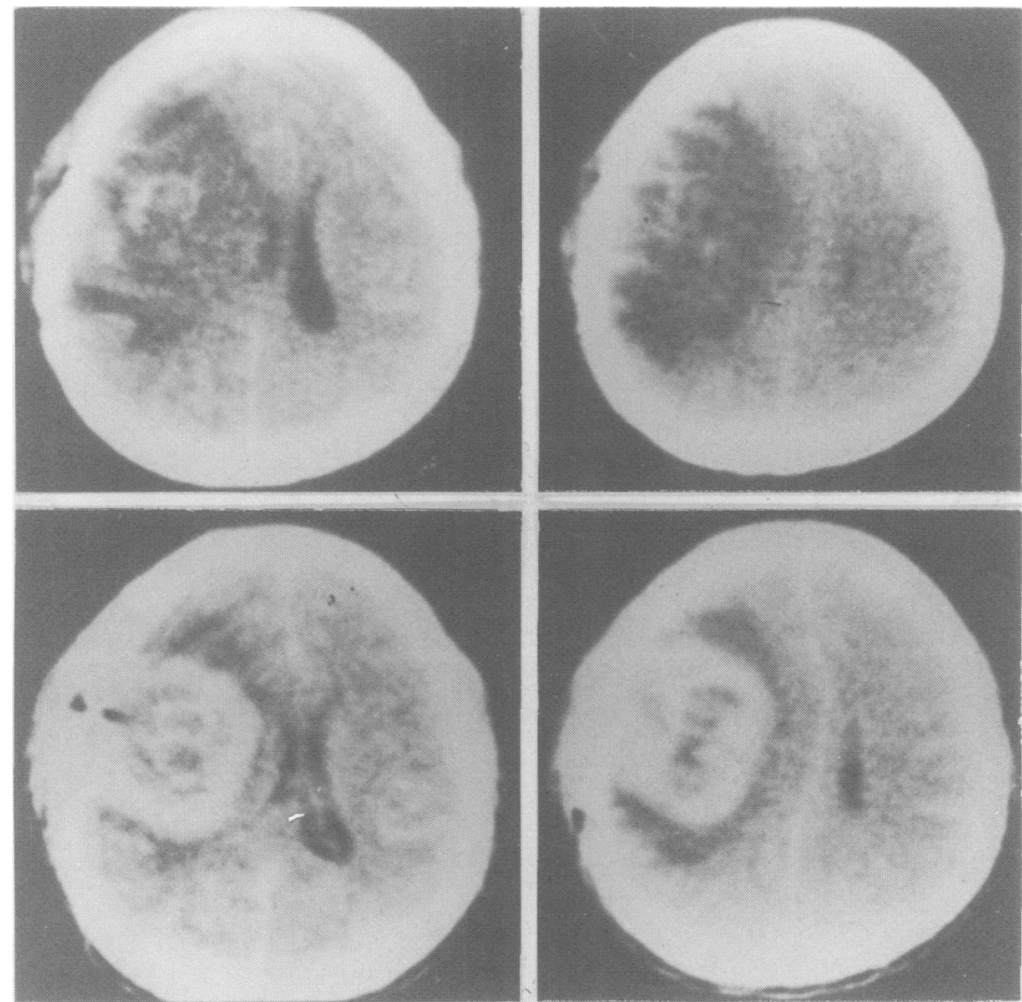

Fig 1 (a) CT scan of Case 1 showing a left fronto-parieto-temporal lesion with heterogenous density and a mass effect (upper row). The mass exhibited a ring enhancement with surrounding oedema in the contrast study (lower row).

weakness. Open brain biopsy was then performed on 9 and 15 September. During these operations, no definite abnormality in the cerebral cortex was found. Both biopsy specimens showed similar inflammatory changes. The patient continued to have headache, progressive weakness of right extremities and episodes of right facial twitching. Follow-up CT on 23 September showed increased size and density of the mass lesion and increased surrounding oedema (fig 1, a). In the contrast study, there were ring and heterogenous enhancements. Two months after admission, the patient developed a flurry of generalised convulsions that were followed by coma, right hemiplegia and dilatation of left pupil. Emergency CT scan disclosed a further enlargement of the lesion. The patient expired 2 days later. Necropsy revealed significant pathological findings limited to the brain. The cerebral hemisphere was diffusely oedematous, greater on the left side with a transtentorial herniation of the left uncus, hippocampus and diencephalon. On cross sections, old and recent necroses and haemorrhages with oedema were seen in decreasing severity from the frontal to the occipital lobe. The most striking finding was extensive haemorrhagic necrosis confined to the subcortical white matter with relative preservation of the cerebral cortex in the fronto-parieto-temporal regions (fig 2). Microscopic examination revealed extensive demyelination mixed with necrosis, ball and ring haemorrhages, fibrinoid necrosis of blood vessels and mild perivascular lymphocytic infiltration. No viral inclusion body or neoplastic cells were seen. The necropsy diagnosis was acute haemorrhagic leucoencephalitis.

Case 2 The patient was a 24 year old man who was admitted to the hospital on 19 November, 1984 with a 3 weeks' history of headache, blurred vision and poor memory. About one month prior to the admission, he developed upper respiratory tract infection with nasal obstruction, rhinorrhoea and low grade fever. One week later, he began to suffer from blurred vision, memory impairment and poor attention. His headache became intense and persistent 2 days before admission. General examination on admission revealed body temperature $36 \cdot 8^{\circ} \mathrm{C}$, blood pressure $120 / 80 \mathrm{mmHg}$ and pulse rate $100 / \mathrm{min}$. The neck was supple. There was no papilloedema. Neurological examination showed confusion to time, place and person, impairment of immediate memory and left inferior quadrantanopsia. Deep tendon reflexes were moderately increased on both sides and the plantar responses were flexor. EEG disclosed intermittent theta and delta waves over the right parieto-temporal areas. CT scan showed abnormal attenuation with a mass effect at the right parieto- 


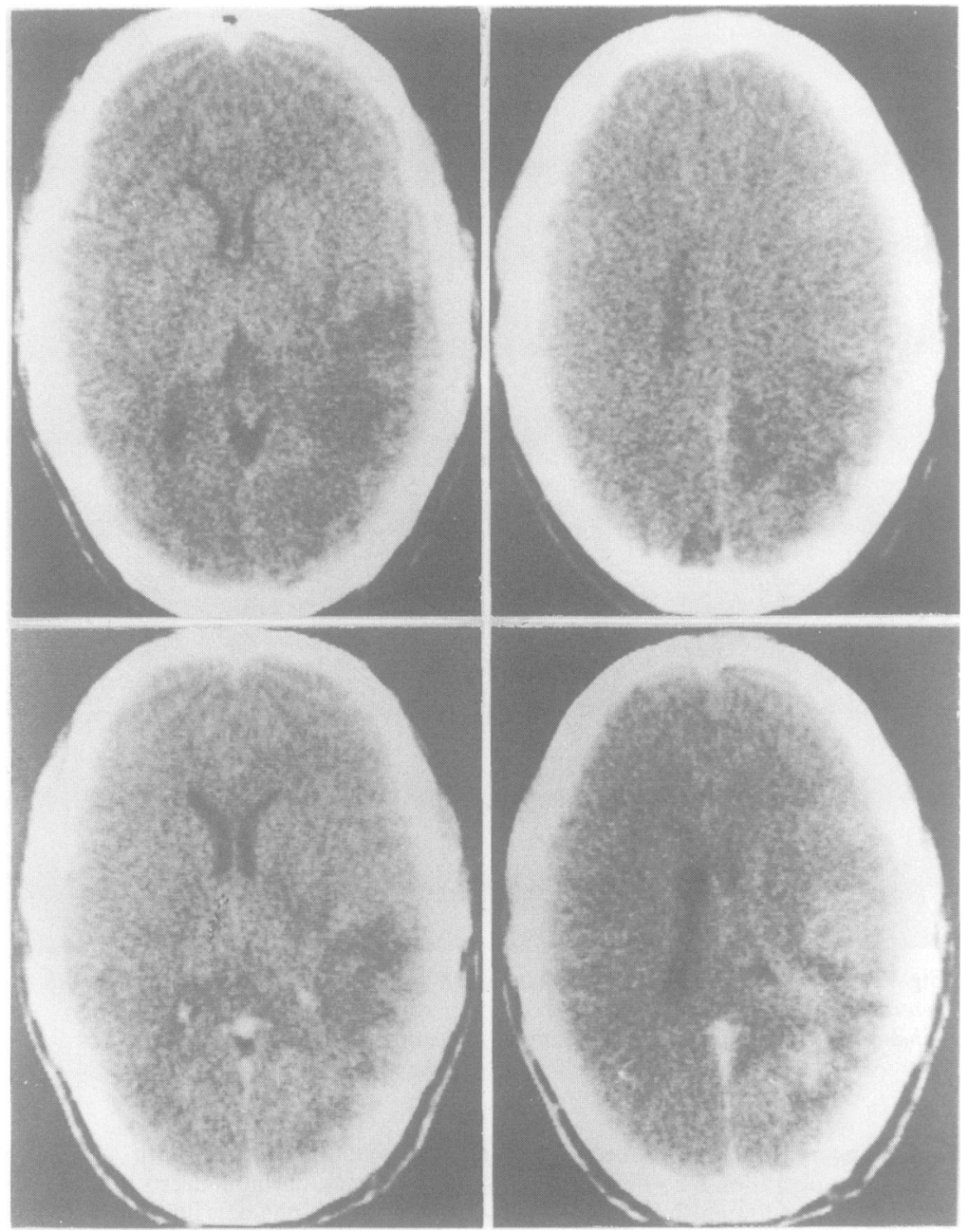

Fig 1 (b) CT scan of Case 2 showing irregular attenuation in right parietotemporal lobes (upper row) and heterogenous enhancement (lower row).

temporal regions that showed a heterogenous enhancement after contrast medium infusion (fig 1, b). The patient was treated with steroid and hyperosmolar agents. Cerebral angiography confirmed a mass lesion without pathological vessels. Decompressive temporal lobectomy was performed on 10 December, 1984. Examination of the removed tissues revealed demyelination in the subcortical white matter with sparing of U-fibres, prominent perivascular lymphocytic cuffings and perivascular demyelination with relative preservation of axons. Multiple scattered petechial haemorrhages were also found. The pathological diagnosis was haemorrhagic and demylinative leucoencephalitis, most likely acute haemorrhagic leucoencephalitis.

The patient's symptoms had gradually subsided 2 weeks after the operation, but seizures developed 4 months later. The seizures were usually preceded by hallucinations of hear- ing bugs humming and sometimes smelling a queer odour for several seconds followed by generalised convulsions. EEG showed focal epileptiform activity over right centrotemporal areas. The seizures were well controlled with phenytoin. Follow-up CT demonstrated in the right temporal lobe an area of decreased density without contrast enhancement.

\section{Discussion}

The diagnosis of acute haemorrhagic leucoencephalitis in the two cases reported here was confirmed pathologically, one at necropsy and the other from a large biopsy specimen. In Case 2, the histological picture was more typical of acute hae- 


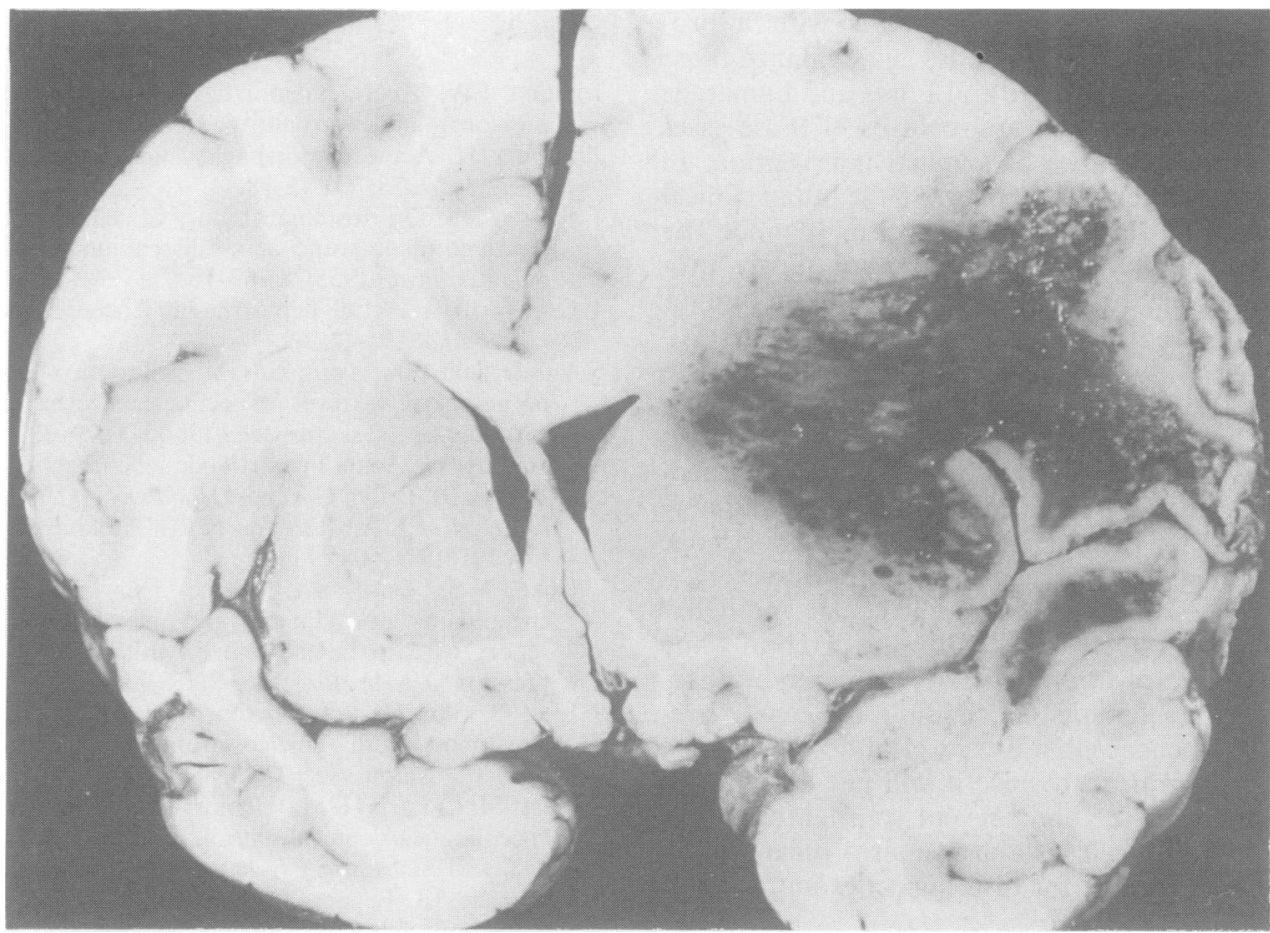

Fig 2 Coronal section of the cerebrum from Case 1 shows an extensive haemorrhagic necrosis in the left fronto-parieto-temporal white matter with relative preservation of the cerebral cortex.

morrhagic leucoencephalitis but the location of the lesion in the temporal lobe raised the possibility of herpes simplex encephalitis which was, however, easily ruled out because the cerebral cortex was free of inflammatory changes, viral inclusions or neuronophagias. Though there were some differences in the degree of severity, both of our cases fulfilled the diagnostic criteria advocated by the previous investigators. ${ }^{1-356}$

What made these two cases unusual were their clinical courses. Typical acute haemorrhagic leucoencephalitis is characterised by an abrupt onset, a rapidly progressive clinical course in a matter of days and a rapidly fatal outcome. Our two cases did not follow this pattern. The onset was insidious in both cases. Case 1 had a chronic course lasting 3 months and died of brainstem compression secondary to transtentorial herniation. Case 2 had a subacute course for one and half months and recovered.

Several similar cases have been reported in the literature. Reich et al ${ }^{12}$ reported a subacute case of presumed acute haemorrhagic leucoencephalitis with eventual recovery. The initial CT and a brain biopsy, which might have been performed too early, showed no abnormality. CT scan 2 weeks later revealed large areas of decreased attenuation involving the white matter of both frontal lobes. Kristiansen $e a^{11}$ reported two patients who survived following decompressive craniotomy and the diagnosis of acute haemorrhagic leucoencephalitis was made by brain biopsy. Coxe and Luse ${ }^{7}$ showed two biopsy-proven cases with a long survival following surgical decompression. One patient was alive two and a half years after the surgery but was left with mild weakness of left upper extremity, left homonymous hemianopsia and a seizure disorder. The second patient survived for nearly one year exhibiting a chronic relapsing course. A second craniotomy was performed 7 months after the initial operation but the procedure did not prevent further neurological deterioration that eventually appeared to involve the brainstem. The patient of Lamarche et al ${ }^{10}$ survived 9 months following a partial left frontal lobectomy but remained in a mute state with ballistic movements and intractable seizures. Those reports seem to indicate that surgical decompression may prevent a fatal outcome in some patients with acute haemorrhagic leucoencephalitis but a progressive neurological deterioration can also occur in some others. The latter observation suggests that a progressive chronic form of acute haemorrhagic leucoencephalitis may exist.

Our Case 1 was the first necropsy proven case of 
acute haemorrhagic leucoencephalitis with a progressive chronic course. Our Case 2 is similar to those of Kristiansen et al ${ }^{11}{ }^{1}$ Coxe and Luse ${ }^{7}$ and Lamarche et al. ${ }^{10}$ However, the natural courses of those cases are not known because of surgical intervention. In contrast to the previous reports, ${ }^{1-6}$ the initial clinical presentation of our cases was surprisingly mild. The clinical courses of both patients were slowly progressive and were highly suggestive of a brain tumour clinically.

The present and previous reports indicate that subacute and chronic forms of acute haemorrhagic leucoencephalitis may exist and the prognosis is not always grave. These forms of acute haemorrhagic leucoencephalitis may pose a diagnostic challenge and may be difficult to distinguish from a haemorrhagic tumour or a haemorrhagic infarct. However, a unilateral hemispheric mass with irregular and heterogenous enhancement localised in the cerebral white matter with preservation of the overlying cerebral cortex is a characteristic CT finding for acute haemorrhagic leucoencephalitis. Surgical decompression is highly recommended since it will not only relieve intracranial pressure and prevent transtentorial herniation, but also will establish a firm diagnosis by obtaining a specimen for histological examination.

Dr Shaw was a visiting Professor from the Department of Pathology, University of Washington, Seattle, Washington, USA.

The authors are indebted to Ms YL Chiang for preparation of the manuscript.

\section{References}

1 Hurst EW. Acute haemorrhagic leucoencephalitis: a previously undefined entity. Med J Aust 1941;28:1-6.

2 Lander H. Acute hemorrhagic leucoencephalitis. Aust Ann Med 1958;7(1):55-68.

3 Russell DS. The nosological unity of acute hemorrhagic leucoencephalitis and acute disseminated encephalomyelitis. Brain 1955;78:369-76.

4 Crawford T. Acute hemorrhagic leucoencephalitis. $J$ Clin Pathol 1954;7:1-9.

5 Vanderfield GK, Tompkins M, Jelihovsky T. Clinicopathological features of acute hemorrhagic leucoencephalitis. Aust Ann Med 1960;9(1):29-33.

6 Gosztonyi G. Acute hemorrhagic leucoencephalitis. In: Vinken PJ, Bruyn GW, eds. Handbook of Clinical Neurology, Vol 3. Amsterdam: North-Holland Publishing Co, 1978;587-604.

7 Coxe WS, Luse SA. Acute hemorrhagic leucoencephalitis: A clinical and electron-microscopic report of 2 patients treated with surgical decompression. J Neurosurg 1963;20:584-96.

8 Litel G, Ehni G. Acute hemorrhagic leucoencephalitis: Treatment with corticosteroids and dehydrating agents. $J$ Neurosurg 1970;33:445-52.

9 Hart MN, Earle KM. Hemorrhagic and perivenous encephalitis: A clinical-pathological review of 38 cases. J Neurol Neurosurg Psychiatry 1975;38:585-91.

10 Lamarche JB, Behan PO, Segarra JM, Feldman RG. Recurrent acute necrotizing hemorrhagic encephalopathy. Acta Neuropathol (Berl.) 1972;22:79-87.

11 Kristiansen K, Harkmark W, Cohen MM. Acute hemorrhagic encephalitis. Neurology 1956;6:503-9.

12 Reich H, Lin SR, Goldblatt D. Computerised tomography in acute hemorrhagic leucoencephalopathy: a case report. Neurology 1979;29:255-8. 